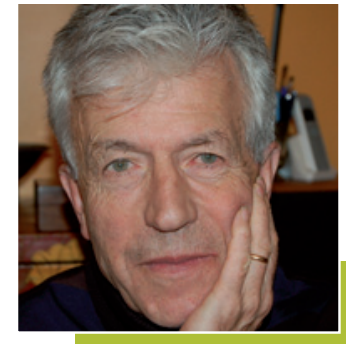

Michel COTTEN

Trésorier-Payeur général honoraire

\title{
La réforme territoriale : une réforme qui avance
}

\begin{abstract}
Moł5-clés: finances locales - structures locales - décentralisation - régions - métropoles intercommunalités - maîtrise des dépenses locales
\end{abstract}

\section{Les réformes successives intervenues dans le domaine des collectivités territoriales vont dans le sens d'une simplification et d'une modernisation progressive, réussies pour les structures, inabouties sur les compétences, incomplètes sur les finances malgré la maîtrise des dépenses.}

Catégorie d'EPCl créée par la loi Sarkozy de décembre 2010, reprise et amplifiée par la loi MAPTAM de janvier 2014. La métropole de Lyon est la seule à bénéficier du statut de « collectivité territoriale spécifique » en raison de l'élection au suffrage universel en 2020 des conseillers communautaires. La Corse est aussi une collectivité territoriale à statut spécifique ;

l'île de Saint-Barthélemy également. En vertu du traité signé avec la Suède au moment de la cession, la France s'est engagée à ne jamais créer d'impôt dans ce territoire.

Cet article est paru dans le $n^{\circ} 2$ - mai 2016 de la revue llissos que nous remercions.

a réforme territoriale a donné lieu depuis des années à un travail législatif continu et le quinquennat qui s'achève a franchi quelques étapes décisives. Au-delà des critiques sur le «millefeuille » et sur le laxisme des gestions locales, se profile lentement le nouveau visage d'un cadre territorial modernisé.

La création de 12 métropoles ${ }^{1}$ est un acte de grande portée et la mise en place de la métropole de Lyon dès 2015 en est le meilleur exemple. La métropole de Paris, paralysée par des tensions internes, ne pourra rester longtemps à l'écart de ce mouvement. Dans le même temps, la réforme des intercommunalités a fait du chemin et va conduire fin 2016 au regroupement de $98 \%$ des communes au sein d'environ 1000 à
1200 communautés. La création de régions nouvelles est venue récemment couronner ce programme. Le département, lui, est resté à l'écart de ces bouleversements; cela dit toutes les métropoles, à l'instar de Lyon, ont vocation à exercer les compétences départementales dans leur périmètre.

Ces efforts de simplification se sont heurtés à la définition des compétences et aux mauvaises habitudes des financements croisés ; si la compétence générale reste réservée au bloc communal, des exceptions complexes vont ouvrir un vaste champ à des tractations interminables entre les collectivités. 
Des efforts substantiels ont été faits pour maitriser l'évolution des finances locales contrairement à une opinion répandue, mais ces efforts n'ont pas amélioré la lisibilité d'un système financier qui reste inaccessible au non-spécialiste. Enfin beaucoup d'efforts restent à faire pour assurer la mutualisation effective des moyens; les effectifs ont continué à croître de $2 \%$ par an de 2002 à 2013.

Compte tenu du peu d'intérêt porté par l'opinion publique pour ces réformes, l'action en faveur de la démocratisation et la participation demeure bien nécessaire. La mixité homme-femme dans les élections locales a été une étape, mais c'est surtout l'élection au suffrage universel en 2020 dans les communautés de communes qui fera émerger la réalité du changement.

L'expression «millefeuille territorial » a fait fortune. Le jugement sévère sur la gestion financière des collectivités locales est tout aussi répandu. Derrière ces appréciations sommaires, nourries par l'exaspération de beaucoup de contribuables devant la croissance rapide des impôts locaux ${ }^{2}$, se cache un dossier d'une grande complexité, qui a fait l'objet depuis 30 ans de réformes continues.

Cette note a pour objet de présenter une vision de ce long cheminement. À travers les alternances et de nombreuses remises en cause, ces réformes ont permis de progresser vers la modernisation et la simplification du cadre territorial. II y faudra encore du temps et de l'obstination. Ce processus peut paraître étrange comparé aux changements rapides opérés ailleurs. Mais on voit se dégager progressivement l'image d'une administration nouvelle reposant sur les régions et les agglomérations.
Après les lois Defferre, acte 1, et les textes Raffarin, notamment la réforme constitutionnelle de 2003, acte 2, il était logique pour le candidat Hollande de proposer un acte 3, en rupture bien sûr avec les mesures prises par son prédécesseur, Nicolas Sarkozy.

À La Rochelle, lors de l'université d'été du PS d'août 2012, Mme Lebranchu, ministre de la décentralisation, énonçait un vaste programme législatif, comportant notamment un projet de loi visant à faire une plus grande place au citoyen. En octobre au Sénat, le Président de la République fraîchement élu confirmait le projet. Et puis tout a changé.

Dans ce domaine comme dans d'autres, les réalisations du Président ne correspondent pas aux engagements pris par le candidat, en particulier celui de maintenir les dotations de l'État au niveau existant. Cela dit, à un an de la fin du quinquennat le bilan est loin d'être négatif et paradoxalement on observe une grande continuité entre la politique du président Sarkozy, marquée notamment par la loi du 16 décembre 2010 sur la réforme des collectivités territoriales et les mesures effectivement prises sous la présidence Hollande. Deux inspirations communes : la fin d'un certain « romantisme » de la décentralisation au profit de l'efficacité ; l'acceptation de solutions diversifiées adaptées à des situations locales différentes. Paradoxalement, jusqu'à une époque récente, la décentralisation se faisait suivant des méthodes jacobines. Et pourtant, depuis 2003, l'article $1^{\text {er }}$ de la Constitution affirme que l'organisation de la France est décentralisée!

\section{La fin du « millefeuille » est désormais en vue}

Autour des années 60, la plupart des pays européens ont réduit drastiquement le nombre de leurs communes. Tétanisés par les associations d'élus et le Sénat ( le grand conseil des petites communes de France »), les gouvernements français successifs n'ont pas osé attaquer de front le sujet; la France s'est couverte de syndicats spécialisés, puis à vocation multiple. Et en avant pour le millefeuille.

\section{A. Au 1er janvier 2016, treize métropoles ont été créées, Paris compris}

Une étude de BPI-France a établi que les agglomérations ayant, selon la loi MAPTAM (Moderni- sation de l'action publique et affirmation des métropoles), vocation à devenir des métropoles (plus de 400000 habitants agglomérés dans des aires urbaines de plus de 650000 habitants) abritaient $39 \%$ de la population française, produisaient $51 \%$ du PIB et $70 \%$ des brevets. De 2000 à 2010, la croissance annuelle de ces agglomérations a été de 1,6\% en moyenne, soit un demi-point de plus que la moyenne nationale.

La loi du 27 janvier 2014 dite loi MAPTAM³ a créé dix métropoles de droit commun, dans les agglomérations importantes (Rennes, Bordeaux, Nantes
Désormais, seules les communes ou leurs groupements perçoivent la taxe d'habitation et les taxes foncières, sur les entreprises notamment. Les départements perçoivent encore les taxes foncières; les régions, aucune de ces taxes. Suivant l'art. 72-3 de la Constitution, les ressources fiscales représentent une part " déterminante » des ressources de chaque catégorie de collectivités territoriales.
${ }^{3}$ Loi de modernisation de l'action publique et de l'affirmation des métropoles, du 27 janvier 2014. 
Toulouse, Lille, Rouen, Grenoble, Strasbourg, Montpellier et Brest). C'est une réforme importante car elle correspond aux réalités démographiques et aux nécessités économiques de notre pays ; elle se situe dans la continuité d'une réforme engagée difficilement voici 50 ans avec la loi du 31 décembre 1966, créant d'office quatre communautés urbaines à Bordeaux, Lyon, Strasbourg et Lille-Roubaix-Tourcoing : le Sénat laissa faire, ne s'intéressant qu'aux petites communes rurales. À ces métropoles "MAPTAM », il convient d'ajouter la métropole de Nice, créée sous le régime de la loi "Sarkozy » du 26 décembre 2010.

Des solutions spécifiques ont été retenues pour les trois plus grandes agglomérations : Paris, Lyon et Marseille.

Après un demi-siècle d'existence, la Communauté urbaine de Lyon est devenue le 1er janvier 2015 la "Métropole de Lyon», une vraie " collectivité territoriale » pleinement compétente en matière de transports urbains, de développement économique, d'urbanisme, de logement, de gestion de l'environnement mais aussi d'action sociale. Dans le périmètre de la métropole, les compétences du département du Rhône en la matière sont transférées à la métropole, et intégrées aux autres compétences. Une politique de la ville démocratique et efficace suppose que les politiques des transports, de l'aménagement, de I'urbanisme, du logement, mais aussi de l'action sociale soient placées ensemble sous l'autorité d'exécutifs élus. Le Grand Lyon va faire la démonstration que la suppression du département dans les agglomérations est non seulement souhaitable mais possible. En 2020, le conseil communautaire du Grand Lyon sera effectivement élu au suffrage universel, ce qui découle du statut de Collectivité territoriale.

À Marseille-Aix, le changement s'est révélé plus difficile à mettre en œuvre en raison de la complexité du territoire, concernant près de 2 millions d'habitants regroupant deux Communautés urbaines et plusieurs $\mathrm{EPCl}^{4}$, et aussi faute d'un leader aussi reconnu que Gérard Collomb à Lyon. Intelligemment, dans la préparation, l'accent avait été mis sur le rôle que pourrait jouer la métropole pour résoudre les problèmes des transports, plaie des Marseillais.

L'augmentation des concours financiers, notamment à travers le contrat de Plan, a permis de vaincre certaines réticences (aixoises, essentiellement) et le 19 février 2016 le Conseil constitutionnel a considéré que la composition du conseil communautaire ne violait pas le principe de l'équitable représentation des citoyens.

\section{B. Paris n'aura qu'une métropolette}

Les maires de l'agglomération parisienne ont eu raison de l'ambitieuse structure définie par la loi MAPTAM. La Métropole du Grand Paris (MGP), $\mathrm{EPCl}$ à statut particulier que préside M. Ollier, n'est plus après le long travail de démolition mené par les élus franciliens de base et la maire de Paris, Mme Hidalgo, qu'une coquille à peu près vide, ce qu'a acté la loi NOTRe ${ }^{5}$. Dès son élection, la nouvelle présidente de la région Île-de-France, Mme Pécresse, s'est jointe au chœur des opposants, demandant même la suppression de cette structure dérisoire.

Dans un récent Journal du Dimanche (JDD), M. Devedjian, président du Conseil départemental des Hauts-de-Seine, et opposant de la première heure à la création d'une Métropole du Grand Paris, se montre satisfait du résultat: "La MGP, quelle adresse, quel $n^{\circ}$ de téléphone? On ne tire pas sur un corbillard... »). Effectivement, la MGP n'a pas de compétence propre en matière de transports urbains, d'urbanisme et de développement économique ni même de tourisme. Comme les départements de la petite couronne gardent toutes leurs compétences dans le périmètre de la MGP, à la différence de ce qui a été fait à Lyon, on est tenté de lui donner raison. Une des premières décisions du bureau de la MGP a été de verser des avances à hauteur de $3 \mathrm{Md} €$ environ aux 19 groupements de communes constitués en son sein ; ceux-ci ont la réalité du pouvoir.

Le Grand Lyon va pouvoir jouer dans la même cour que Manchester ou Milan, métropoles européennes. En revanche, la MGP, en raison notamment de son déficit institutionnel, va continuer de perdre du terrain par rapport à ses rivales européennes Londres et Berlin et finir par sortir de la catégorie des "villes-monde», pour entrer dans celle des «villes-musées » aux côtés de Rome.

Les Jeux olympiques de 2024 et l'exposition universelle de 2025 auraient pu servir de rampe de lancement à la métropole nationale; eh bien non, la Ville de Paris entend préparer seule les $\mathrm{JO}$, alors que la loi donne expressément compétence à la MGP pour ce faire. C'est pourtant l'une des rares compétences clairement attribuée à la MGP. Ce problème de gouvernance n'échappera certainement pas le moment venu aux juristes du $\mathrm{ClO}$. 
C. D'ici la fin de l'année en cours (2016), près de la moitié des groupements de communes auront fusionné

L'achèvement des travaux de rationalisation de la carte des intercommunalités est également en bonne voie. Les élus avaient jusqu'au 31 mars 2016 pour amender les projets de cartes préparés par les préfets; assez souvent ces amendements vont au-delà des propositions préfectorales.

Fin 2016, plus de 98 \% des communes françaises seront regroupées au sein de communautés de communes, de communautés d'agglomération, de communautés urbaines ou de métropoles, au nombre d'un millier environ, qualifiés d'EPCI.

Les syndicats de communes (au nombre de 9428 ) et les syndicats mixtes (306) sont également appelés à se fondre dans ces EPCl élargis.

On peut faire confiance à la nouvelle secrétaire d'État aux collectivités locales, Mme Grellier, ancienne vice-présidente de l'Association des Communautés de France et militante de l'intercommunalité, pour atteindre l'objectif.

Pendant que la plupart des pays européens (Allemagne, UK, Belgique, etc.) réduisaient drastiquement le nombre de leurs communes, la France mettait timidement en place des syndicats à vocation unique puis multiple étroitement dépendants des communes de base. La loi Chevènement de 1999 portant " Renforcement et simplification de la coopération intercommunale " avait donné un coup de fouet décisif à la création des communautés de communes en milieu rural comme dans les agglomérations urbaines, ceux-ci disposant d'une fiscalité additionnelle ou d'une taxe professionnelle unique et bénéficiant de dotations renforcées au titre de la DGF-Intercommunalité.

Il fallu attendre la loi Sarkozy de 2010 pour abandonner le « volontariat encouragé » et passer à un plan de regroupement, tenant compte des préférences des communes, mais pouvant se terminer par une décision du préfet non conforme aux souhaits exprimés.

La loi MAPTAM a rappelé l'objectif et donné plus de pouvoirs encore aux préfets pour aboutir dans des délais stricts à des solutions rationnelles, éventuellement en passant outre aux oppositions locales injustifiées.

L'opération, menée sans trop de bruit, car les journalistes ne montrent aucune curiosité pour les trains qui arrivent à l'heure, va aboutir selon la nouvelle secrétaire d'État à une réduction de $40 \%$ du nombre de groupements de communes à fiscalité propre : il ne restera qu'environ mille groupements.

La loi de 2010 avait fait de la « mutualisation des services (et de leurs personnels) » un axe fort de cette rationalisation; la loi MAPTAM a rendu contraignant l'exercice : non seulement tous les personnels correspondant à une compétence transférée à l'EPCl doivent suivre cette compétence là où elle s'exerce désormais : la mutualisation des services communs hors transferts est également encouragée.

Les conditions vont être réunies pour qu'aux prochaines élections municipales (2020) se produise un double basculement. Les conseillers communautaires seraient dorénavant élus directement au suffrage universel, et non plus simplement fléchés, comme aux élections municipales de 2014. Les EPCl et les métropoles deviendraient ainsi, le temps aidant, plus légitimes que les communes.

Les EPCI pourraient alors devenir les seules autorités fiscales du «bloc communal», les communes recevant désormais des dotations budgétaires, un peu comme les actuels arrondissements de Paris, Lyon et Marseille.

\section{Des régions plus grandes mais sans moyens adaptés}

Par ailleurs, a eu lieu dans des conditions improvisées un regroupement des 23 régions (métropolitaines) dotées d'assemblées et d'exécutifs élus depuis 1986, en 10 nouvelles régions. II n'y a plus qu'une seule Normandie, mais la Bretagne reste privée de sa capitale historique, Nantes; pourtant le "Gwen ha du » (drapeau breton) flotte sur l'hôtel du département de Loire-Atlantique depuis 2002.

La grande presse a attaché une importance excessive à cette opération spectaculaire, tandis que le remodelage de la carte des intercommunalités passait presque inaperçu.

Petites ou grandes, les régions restent des nains financiers.

Rien à dire à ce stade des départements plusieurs fois menacés de suppression et finalement conservés tels quels, sauf à Lyon. Les tergiversations de l'exécutif les ont plutôt renforcés. 


\section{La tentative de " clarification des compétences" n'a pas abouti}

Dans un premier temps - loi MAPTAM du 29 janvier 2014 - le législateur avait rétabli la clause de compétence générale, enlevée par la loi Sarkozy de 2010 aux régions et aux départements, c'est-à-dire codifié la confusion des responsabilités.

Après réflexion, la loi NOTRe a repris le dispositif de 2010. En clair, les régions et les départements ne peuvent intervenir qu'au titre des compétences listées par la loi. Seules les communes continuent de bénéficier de la clause de compétence générale. Une commune ou un EPCl peut intervenir dans tous les domaines, sauf ceux où la loi a donné compétence exclusive à la région ou au département.

Vu de plus près, ce n'est pas si simple.

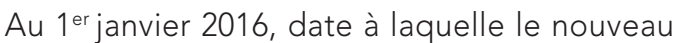
dispositif s'applique, le droit est le suivant:

- Si aucun texte ne désigne le responsable d'une compétence, en bonne logique, seules les communes et leurs groupements, dotés d'une compétence générale, pourraient l'exercer, à l'exclusion des collectivités à compétences d'attribution : régions et départements. Un commentateur officiel, le ministre de l'intérieur, considère néanmoins que les départements pourront continuer d'intervenir en matière de liaisons aériennes et de parcs naturels régionaux, en raison de leur compétence en matière touristique !

- Certaines compétences sont strictement exclusives et ne peuvent pas être déléguées, comme l'élaboration du Schéma régional de développement économique évidemment mais aussi la gestion des fonds structurels européens que l'État leur a confiés. Toutefois le schéma devra être élaboré conjointement avec la ou les métropoles existant dans la région. De toute façon, le schéma devra être approuvé par le préfet. L'État est de retour.

- Le titulaire officiel de la compétence peut toujours en déléguer l'exercice, dans le cadre d'une convention. La région est en principe seule compétente pour définir et octroyer les aides à la création et extension d'activités, comme pour aider les entreprises en difficulté, mais elle peut déléguer l'exercice d'une partie de ses attributions à un département, une commune ou un EPCl.

Les communes et les EPCl sont seuls compétents pour accorder les aides à l'immobilier d'entreprise, mais rien ne leur interdit de passer des conventions avec la région pour la mise en œuvre. Enfin si les départements n'ont plus de compétence en matière économique, ils peuvent continuer d'intervenir au titre de la solidarité territoriale et compléter les aides de la région dans le domaine agricole...

- Certaines compétences, par nature transversales, restent "partagées ». L'article 104 de la loi NOTRe en liste cinq : la culture, le sport, le tourisme, les langues régionales, l'éducation populaire. Mais suivant les "instructions du Gouvernement du 22 décembre 2015 », cette liste n'est pas exhaustive ; il faudrait rajouter au moins l'eau et le numérique.

- Enfin, la loi MAPTAM prévoyait que les collectivités pouvaient désigner un « chef de file » pour exercer certaines compétences, ce qui correspond à un mode conventionnel de partage des compétences. Les modalités en seront fixées dans des «Conventions territoriales d'exercice des compétences».

Les projets de conventions devront être préalablement débattus au sein des "Commissions territoriales d'action publique » créées par la loi.

C'est au sein de cette instance que sera défini le projet de schéma régional.

La région pourra être chef de file dans huit domaines : aménagement et développement durable, protection de la biodiversité, climat air et énergie, développement économique, soutien à l'innovation, internationalisation des entreprises, aménagement du territoire, soutien à l'enseignement supérieur.

Le département pourra être chef de file en matière d'aide et d'action sociale, d'autonomie des personnes, de solidarité des territoires.

La commune ou le groupement pourront de leur côté être chef de file pour la mobilité durable, les services publics de proximité, l'aménagement de l'espace et le développement local.

Ce dispositif a été conservé d'une loi à l'autre, malgré la suppression de la clause de compétence générale pour les régions et les départements, sans doute par négligence.

Par ailleurs, à défaut de supprimer totalement les "financements croisés», qui permettent aux maires habiles de ne faire supporter par leurs communes qu'une partie minime de la charge financière des ouvrages qu'ils s'apprêtent à 
inaugurer, la loi NOTRe fixe des minima de participation : 30 \% si le maître d'œuvre est en même temps chef de file, $20 \%$ dans les autres cas. De nombreuses dérogations sont prévues concernant la rénovation urbaine, la restauration des monuments historiques, les opérations prévues au contrat de Plan... et les travaux réalisés en Corse. Notons que le maître d'ouvrage n'ayant financé effectivement que $20 \%$ des travaux, touchera du Fonds de compensation de la TVA (FCTVA) une allocation calculée sur $100 \%$ des travaux.

L'analyse ci-dessus montre que les bonnes intentions gouvernementales de départ n'ont pas résisté à la discussion parlementaire. La répartition des compétences résultant des lois récentes n'est ni exhaustive ni univoque. Avec un peu d'habileté, les gestionnaires pourront faire presque comme avant. Le leadership de la région en matière économique est affirmé mais ne couvre pas la totalité du domaine ; il pourra être contourné à coups de conventions et de dérogations. Le préfet essaiera d'intervenir mais on lui rappellera vite que les collectivités se gèrent librement. Ce montage juridique complexe ne tiendra sans doute pas la route longtemps et il faudra reprendre la question, au vu de l'expérience.

\section{Les finances des collectivités locales sont en voie d'être maîtrisées}

\section{A. La décentralisation financière reste limitée en France}

Après trente ans de "décentralisation ", les budgets des collectivités locales françaises et des organismes rattachés ne représentent que $19 \%$ des dépenses publiques. Dans un pays comme le Danemark, par exemple, le chiffre correspondant est de $34 \%$. En effet, dans la plupart des pays européens les dépenses d'enseignement primaire et de sécurité figurent dans les budgets locaux.

L'État reste très présent dans la vie financière quotidienne des collectivités locales.

Les impôts locaux restent assis et recouvrés par des fonctionnaires d'État; le comptable de la collectivité est également un fonctionnaire d'État, I'ancien percepteur, trésorier. Les maires français n'ont pas de carnet de chèques... Tous les mois à date fixe, l'État verse aux collectivités locales, sur leur compte au Trésor public, le seul qu'elles ont le droit d'avoir, des douzièmes de fiscalité et de DGF6, ce qui facilite la tâche des gestionnaires locaux.

Les collectivités locales déposent obligatoirement leurs fonds au Trésor public, y compris ceux provenant d'emprunts contractés mais non encore utilisés. L'État dispose ainsi gratuitement d'un volant de plus de $20 \mathrm{Md} €$. Les communes belges, par exemple, déposent leurs fonds libres au Crédit communal de Belgique.
Les préfets, au titre du contrôle budgétaire, veillent avec le concours des Chambres régionales des comptes au strict respect de l'équilibre des budgets et surtout des comptes. Maintenant comme avant la décentralisation, en cas de dérapage ils disposent des plus larges pouvoirs pour rétablir l'équilibre au plus tôt. Ces pouvoirs sont effectivement mis en œuvre. Environ une douzaine seulement de communes (sur 36 744) font tous les ans l'objet d'une procédure de redressement. La notion de "commune en faillite » n'est qu'une expression journalistique.

Du point de vue de l'analyse financière, la situation des collectivités locales reste très saine, même après les premiers coups de rabot portés aux dotations de l'État (réduction de la DGF de 12,5 milliards sur quatre ans).

Les collectivités locales françaises réalisent un montant d'investissement important : $55 \mathrm{Md} €$ en 2015 , soit environ $60 \%$ de l'investissement public, qu'elles autofinancent largement, comme les entreprises. Seules les régions, désormais dépourvues de toute fiscalité modulable, ne dépassent pas le taux de $20 \%$.

L'emprunt ne peut financer que des dépenses d'équipement, alors que l'État paye ses fonctionnaires en partie sur fonds d'emprunt. Le recours à l'emprunt reste modéré, au point que durant la période récente (1996-2002), les remboursements d'emprunts ont dépassé chaque année le montant des emprunts nouveaux! Pendant six ans, les collectivités locales se sont désendettées.
${ }^{6}$ Dotation globale de fonctionnement. Créée en 1979, cette dotation (33,1 Md€ pour 2016) regroupé plusieurs transferts financiers de l'État aux collectivités territoriales Elle est attribuée en fonction de critères objectifs et non de projets ; elle est libre d'emploi ; indexée initialement sur le produit net de la TVA, elle a fait

l'objet de réductions massives depuis 2014. 
Dès lors, le montant de la dette des collectivités locales reste globalement faible : il ne représente que $9 \%$ environ de la dette publique totale au sens du traité de Maastricht.

\section{B. Le rythme de croissance des dépenses du secteur local vient d'être divisé par 2}

Depuis longtemps, la direction du budget se demandait comment ralentir puis inverser la courbe des dépenses locales, tout en respectant le principe constitutionnel de la libre administration des collectivités locales et sans provoquer la colère des associations d'élus.

Depuis 30 ans, les dépenses locales ont constamment augmenté plus vite que le PIB ; la part des collectivités locales est ainsi passée de $8,6 \%$ à $11,8 \%$ du PIB soit $+3,2$ points. Suivant cette étude, la « décentralisation des compétences » n'explique que la moitié de la progression. Hors décentralisation, les dépenses locales ont augmenté de 3,2 \% par an en volume, en moyenne depuis 30 ans.

Suivant le travail auquel s'est livré le ministère des finances (annexe jaune du projet de loi de finances pour 2016), l'essentiel de cette progression est lié à celle des dépenses de fonctionnement et singulièrement à l'augmentation des dépenses de personnel, qui expliquerait 83,6\% de la progression. Contrairement à ce qu'aimeraient croire beaucoup d'élus, les dépenses d'investissement ont crû deux fois moins vite que les dépenses de fonctionnement.

Les collectivités ont donc été conviées à «s'associer» à l'effort d'assainissement des finances publiques décidé par le Gouvernement.

Le 29 avril 2015, dans le cadre du « programme de stabilité 2014-2017», appelé à être notifié aux instances européennes, le Gouvernement fixa à $50 \mathrm{Md} €$ le montant des économies à faire sur le budget de l'État. La contribution des collectivités a été arrêtée à $11 \mathrm{Md} €$ sur trois ans, par tranches de 3,67 Md€ par an, ne représentant après tout que 1,9\% des recettes réelles annuelles.

Dés la première année du programme de réalisation de ce programme, on a observé une décélération globale des dépenses locales, n'affectant pas sensiblement les programmes d'investissement, plus rapide même que celle envisagée dans le programme!

En 2016, la progression des dépenses locales ne devrait pas dépasser 1,2\%, ce qui correspond à l'objectif retenu par la Conférence des finances publiques.

C'est au sein de cette conférence que fut annoncé pour la première fois un Objectif global d'évo- lution des finances publiques locales (ODEDEL), décliné par catégories de collectivités. C'est l'article 11 de la loi de Programmation des finances publiques pour la période 2011-2014 qui a permis de fixer ce taux, révisé chaque année et décliné par groupe de $\mathrm{CL}^{7}$ (bloc communal, départements, régions). Pour l'instant, ce taux reste indicatif et pourrait le rester si aucun dérapage n'est constaté.

Désormais, les CL sont traitées comme les Administrations sociales qui connaissent depuis longtemps déjà une norme d'évolution globale de leurs dépenses (ODAL).

Le nouveau programme de stabilité financière (2016-2018) a confirmé la « trajectoire » du programme précédent en mettant l'accent sur la suppression des normes coûteuses ou inutiles imposées par l'État aux CL; en effet en année pleine les nouvelles normes imposées au secteur local représentaient un surcoût de 1,5 Md€. Une circulaire du 9 octobre 2014 a imposé que le coût de chaque nouvelle norme soit compensé désormais par la suppression d'une norme devenue obsolète.

L'analyse des comptes impactés par ces mesures fait apparaître un net ralentissement global des dépenses locales conforme à l'objectif, l'ajustement s'étant fait principalement par une légère baisse de l'effort d'investissement et de l'autofinancement.

L'année 2015 restera dans les annales comme celle où pour la première fois depuis le début de la Ve République, le montant total des concours aux collectivités locales a globalement baissé d'une année sur l'autre, et ce sans provoquer de drame! Techniquement, c'est la partie forfaitaire de la DGF qui a été amputée, par catégorie de collectivités, proportionnellement aux recettes réelles de fonctionnement.

Le ministre du budget, M. Cahuzac, qui osait beaucoup de choses, avait annoncé sans concertation préalable, au Comité des finances locales, et sans provoquer de troubles majeurs, que les concours de l'État aux collectivités locales seraient réduits de 1,5 Md€. Ce premier pas a encouragé à aller plus loin.

\section{Maîtriser pour de bon les dépenses de personnel, essentiellement dans le " bloc communal ", sera un travail de longue haleine \\ Jusqu'à la loi Defferre de 1984, il n'existait pas de "fonction publique territoriale», mais une liste d'emplois sur lesquels les exécutifs pouvaient recruter assez librement.}

Fin 2015, le nombre d'agents territoriaux, fonctionnaires et non-titulaires dépasse 2 millions. 
De 2002 à 2013, les effectifs locaux ont crû de 2 \% par an en moyenne. Cette augmentation est largement imputable aux groupements de communes. Plus précisément, les transferts de compétences des communes vers les groupements se sont accompagnés trop rarement des transferts de personnels correspondants.

Entre 2002 et 2005, 326000 agents nouveaux ont été recrutés, principalement dans les groupements de communes, qui ont fleuri après la loi Chevènement de 1999. Le fameux principe de mutualisation des moyens humains n'était malheureusement pas une obligation dans la loi Chevènement, et quand on a voulu agir, le mal était fait.

Le personnel des collectivités locales reste encore largement composé d'emplois d'exécution : cantonniers, femmes de ménage, assistantes maternelles, etc.

Trois quarts d'entre eux travaillent dans les communes ou leurs groupements; trois quarts d'entre eux sont des titulaires; trois quarts également relèvent de la catégorie C. Le taux de féminisation atteint $60 \%$.

La rémunération de ces personnels obéit aux mêmes règles que celle de l'État; dans les deux cas, le gel du point d'indice des fonctions publiques depuis 2010, auquel le Gouvernement vient de renoncer à la veille des prochaines élections présidentielles, entretient un sourd mécontentement qui se traduit en particulier par une recrudescence de l'absentéisme.

Enfin, la réforme du mode de recrutement, de formation et de rémunération des cadres dirigeants des collectivités locales ne devrait pas être différée. Le Centre national de formation de la fonction publique territoriale (CNFPT) coûte plus de $555 \mathrm{M} €$ par an ; cet établissement a le monopole de la formation et du recrutement des agents communaux, et ses ressources s'accroissent automatiquement avec l'augmentation des effectifs. Cela n'est pas favorable à l'innovation et à la productivité. Désormais, la formation des administrateurs territoriaux échappe au centre et après une longue bagarre budgétaire, le taux de cotisation obligatoire sur la masse salariale de chaque collectivité est passé de $1 \%$ à $0,9 \%$ ! L'étape suivante serait de soumettre les prestations de formation à la concurrence. La cotisation au CNFPT serait remplacée par un effort budgétaire minimum de formation.

Il a fallu attendre la loi Sarkozy de 2010 pour que la « mutualisation », c'est-à-dire l'utilisation rationnelle des effectifs locaux dans un territoire soit « encouragée », sans toutefois devenir obligatoire.
La loi NOTRe prévoit que les transferts de compétences entre communes et groupements sont assortis de "plans de mutualisation »; le projet initial de réforme de la DGF faisait du « coefficient de mutualisation » un critère important de calcul des dotations communales et intercommunales. Inhibé sans doute par le principe de libre administration, le Gouvernement n'a pas osé, à la différence de pays comme l'Espagne et l'Italie, agir directement sur le niveau des effectifs locaux.

Certains pays de la CEE ont été très loin en agissant directement sur le montant des dépenses de personnel, voire directement sur le niveau des effectifs.

En Italie, les dépenses de personnel local étaient en 2006 supérieures de $17 \mathrm{Md} €$ au montant français ; à la suite de l'application d'un taux de remplacement des départs à la retraite de $20 \%$ seulement, ces dépenses sont devenues inférieures de $8 \mathrm{Md} €$ aux dépenses françaises en 2013 !

En outre, les collectivités qui ne respectent pas la norme s'exposent à de lourdes sanctions comme la réduction des dotations de l'État à concurrence du dépassement, ainsi qu'au gel des embauches.

Même formule au Danemark où le dépassement de la norme entraîne automatiquement une réduction de la dotation de fonctionnement.

L'Espagne a été plus loin encore en décidant des réductions d'effectifs accompagnés de baisses des salaires et d'augmentation de la durée hebdomadaire du travail jusqu'à 37,5 heures.

En France, au total, les dépenses de personnel ont continué à augmenter, quoique beaucoup moins qu'avant.

Cette évolution, encore impensable il y a 10 ans, a pu se faire grâce à la continuité des efforts menés par les gouvernements successifs, en théorie hostiles politiquement, dans le respect formel de la « liberté de gestion des collectivités territoriales » garantie par l'art.72 de la Constitution et moyennant des compensations aux plus fragiles d'entre elles, notamment les départements étouffés par la montée en puissance du RSA.

\section{La solution des autres problèmes financiers des collectivités locales... attendra}

L'obsession de la maîtrise globale des dépenses locales, entretenue par la pression de la Commission européenne, explique en partie que le traitement des autres dossiers n'ait pu aboutir.

La suppression de la taxe professionnelle ${ }^{8}$ a été menée rondement par l'administration Sarkozy. Depuis sa création en 1976, cette taxe n'avait jamais marché ; $5 \%$ des entreprises avaient vu dès la première année suivant « l'enterrement » de la

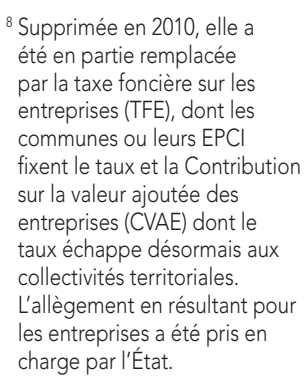
entreprises (TFE), dont les communes ou leurs EPCl fixent le taux et la Contribution sur la valeur ajoutée des entreprises (CVAE) dont le taux échappe désormais aux collectivités territoriales. L'allègement en résultant pour les entreprises a été pris en charge par l'État. 
patente, leur cotisation s'envoler, mais de minimis non curat praetor, la réforme était entrée en application quand même.

Ensuite a commencé la dérive mal contrôlée de cette taxe par rapport aux trois autres (foncières et taxe d'habitation) ; le vote par taxe, malgré le lien obligatoire, mais inadapté, entre la taxe professionnelle et les trois autres n'a pas arrangé les choses. Les entreprises ne votent pas, on peut donc les imposer au maximum; enfin, coup de pied de l'âne, les conseils régionaux tous socialistes, sauf l'Alsace, ont voté en 2005 une augmentation sans précédent de la taxe professionnelle. J'y vois la causa proxima de la réforme de 2010.

Le changement opéré en 2010 a été pour une fois, massif : la taxe professionnelle a disparu pour de bon, remplacée par la Contribution économique territoriale (CET), panier d'impôts, dont la taxe foncière des entreprises, la taxe sur la valeur ajoutée des entreprises, et diverses taxes sur les réseaux. Le total est très inférieur au produit de l'ancienne taxe. Comme d'habitude, l'État a pris la différence sur son budget ; les entreprises voient leur charge allégée et les collectivités leur perte compensée.

Mais le principal point de la réforme est que désormais, les collectivités ne votent plus que le taux de la contribution foncière des entreprises ; le taux de la principale composante de la CET, la $\mathrm{CVAE}^{9}$ est désormais fixé au niveau national à $1 \%$. Le produit national est ensuite réparti entre les groupes de collectivités locales suivant la clé suivante : bloc communal $26,5 \%$, régions $25 \%$, départements $48,5 \%$.

Enfin, et c'est là que le bât blesse, chaque collectivité reçoit une «dotation » calculée au prorata des effectifs de salariés et des valeurs locatives des entreprises présentes sur le territoire ; au final, la répartition est beaucoup plus inégalitaire que celle de la taxe professionnelle. Des critères plus intelligents sont concevables, moins respectueux des intérêts acquis, il est vrai.

Il reste donc un second volet de la réforme à entreprendre, visant à réduire les écarts de dotations par habitant pour les communes et leurs groupements. La mise en place des métropoles et des nouvelles communautés de communes rend moins nécessaire cette réforme : la péréquation se fait tout naturellement par la fiscalité professionnelle unique inscrite aux budgets de ces groupements. C'est de cette péréquation horizontale forte dont les élus de la Métropole du Grand Paris n'ont pas voulu, maire de Paris en tête.

La réforme de la Dotation globale de fonctionnement des communes et de leurs groupements n'aura probablement pas lieu pendant l'actuel quinquennat, les associations d'élus ayant obtenu que les dispositions figurant dans le projet de loi de finances pour 2016 soient « gelées » en 2016.

Les élus ont en effet été effrayés par l'abandon dans le projet de réforme du principe des avantages acquis, et peu rassurés par les garanties en sifflet proposées pour une période intermédiaire : toute commune aurait été assurée de toucher, après la réforme, entre 95 et $105 \%$ des sommes perçues auparavant.

Ils auraient souhaité, conformément aux usages en vigueur depuis 1979, une loi spécifique de réforme de la DGF, après une longue discussion au sein du Comité des finances locales (CFL) ${ }^{10}$.

Cette réforme aura, il est vrai, plus de sens quand la carte des nouveaux EPCl, dont le nombre serait ramené à un millier environ, sera définitivement arrêtée.

Ce gel entraîne du coup le report de la réforme des dotations de péréquation: Dotation de solidarité urbaine et Dotation de solidarité rurale, à laquelle tenaient par contre beaucoup les élus. 2017, année des présidentielles se prête mal à une réforme de grande ampleur. Cet aggionarmento de la DGF du bloc communal devra donc être repris après achèvement de la carte de nouveaux EPCl.

Il y avait, et il y a toujours, au moins trois bonnes raisons de moderniser la DGF du « bloc communal » (21 Md€ sur $50 \mathrm{Md} €$ ) :

- l'utilité de faire périodiquement le ménage dans le fouillis des sous-dotations rassemblées sous le terme de DGF (au moins treize lignes actuellement) ;

- la « contribution des collectivités locales au redressement des finances publiques » se traduit concrètement par un prélèvement massif sur la "dotation forfaitaire », composante principale de la DGF, ce qui déséquilibre le dispositif ;

- la nécessité de mieux prendre en compte les $\mathrm{EPCl}$ (communautés et métropoles) dont le rôle va devenir décisif ; la dotation d'intercommunalité créée par la loi Chevènement avait pour objectif de faire passer une réforme de structures suscitant la méfiance des élus; il s'agit maintenant de définir un régime pérenne des concours de l'État pour des groupements dont les conseils devraient être élus au suffrage universel en 2020, et qui ont vocation à devenir l'échelon local de droit commun.

Les départements, condamnés à mort par le Premier ministre, se retrouvent à peu près intacts en fin de quinquennat, avec au surplus, un ministre départementaliste, Jean-Michel Baylet. Leur principal problème est d'avoir à subir financièrement des décisions prises par l'État en matière sociale, sans concertation le plus 
souvent. La persistance d'un chômage de masse, le vieillissement accéléré de la population et la reconnaissance du handicap les mettent en première ligne avec le RSA, I'APA et la $\mathrm{PCH}$.

Plus de 2,5 millions de foyers émargeaient au RSA en 2014 ; les départements ont eu à débourser $10 \mathrm{Md} €$ à ce titre (+9,3\%). Le Gouvernement décide d'étendre le nombre des bénéficiaires, des revalorisations supplémentaires et une actualisation des barèmes à la hausse, mais ce sont les départements qui payent. C'est en réalité leur seule compétence; un département (67) qui s'est avisé de demander une contrepartie sur la base du bénévolat aux bénéficiaires du RSA, s'est fait taper sur les doigts.

De façon ubuesque, les départements ont été soumis à la contribution au redressement des finances publiques et ont bénéficié au même moment de ressources fiscales supplémentaires par le biais du relèvement du plafond des Droits de DTMO et l'augmentation des droits d'enregistrement et de publicité foncière.

En un sens, la fusion du RSA (Revenu social d'activité) avec la PPE (Prime pour l'emploi) soulage enfin des départements au bord de I'asphyxie. Le Gouvernement songe à recentraliser la gestion du RSA en 2017.

Le principal problème des régions réside dans le décalage entre leurs responsabilités actuelles que la loi NOTRe entend accroître encore et leurs moyens financiers effectifs. La division par 2,5 de leur autofinancement de 2000 (45\%) à 2015 (20\%), ainsi que la progression rapide de leur endettement sont les signes évidents de leur détresse financière. Rappelons qu'en cours de route, les régions ont perdu, au nom de la spécialisation des taxes, l'accès à la taxe d'habitation et aux taxes foncières; depuis la suppression de la taxe professionnelle en 2010, elles reçoivent une quote-part de la CVAE déterminée au niveau national (25\%) sans pouvoir en moduler le taux.

Comme la marge de manœuvre sur la TICPE (taxe sur les carburants) est quasi nulle, il ne reste aux régions que la taxation des cartes grises! La vérité est que les régions (les 13 comme les 26 hier) chargées de conduire le développement économique au niveau local n'ont pratiquement plus aucun pouvoir fiscal. On peut penser que la mise en cohérence viendra.

\section{La démocratisation de la gestion locale}

L'évènement le plus important est l'extension jusqu'aux communes de 1000 habitants des dispositions applicables jusque-là aux plus de 3500 habitants. La réforme s'est appliquée aux élections municipales de 2014. Désormais, I'obligation de parité hommes/femmes descend jusque dans les campagnes.

Les maires de plus de 10000 communes devront compter avec une opposition structurée, alors qu'ils régnaient souvent en maîtres jusque-là. Plus de panachage.

La modification du scrutin cantonal, vieux de plus de 180 ans, relève largement du féminisme, sinon de la théorie du genre : pour réaliser à coup sûr une stricte parité, les candidats se présentent par paires (un homme, une femme), ou comme dirait Peppone, en couple « ambisexuellement composé ».

Le nombre des cantons a été divisé par deux et l'écart-type entre le nombre d'électeurs par canton sérieusement réduit. On ne pourra plus dire que ce sont les hectares qui votent.

Si le Département était maintenu, on pourrait imaginer qu'un jour, lorsque la carte des EPCl sera stabilisée, le conseil départemental soit composé de représentants de ces groupements.

La loi NOTRe a pris acte de l'arrivée d'Internet dans tous les foyers et toutes les mairies. Accompagnant un mouvement spontané, elle prévoit la mise en ligne de toute une série d'informations comme le document établi par le maire à l'occasion du débat d'orientation budgétaire, le compte rendu des séances du conseil municipal, le compte de gestion du maire, son commentaire, les observations de la Chambre régionale des comptes ainsi que la réponse du maire, etc. II ne faut surtout pas brider le zèle des élus dans ce domaine, tout en préservant les droits de l'opposition.

La question du référendum local n'a pas réellement progressé, puisque seul l'exécutif peut soumettre au vote de la population des sujets d'intérêt local.

On a pu croire, après les lois Defferre de 19821984, que la France s'était définitivement convertie à la décentralisation. Dans un premier temps, 
les médias se sont montrés très favorables à cette évolution. Aujourd'hui, ils n'ont pas de mots assez durs pour fustiger le «millefeuille institutionnel», et stigmatiser la gabegie supposée des élus locaux, dont les ronds-points sont devenus le symbole. L'explosion des effectifs locaux, I'absentéisme du personnel et leur faible productivité sont montrés du doigt. Il est clair que la décentralisation n'était pour les médias qu'une mode, voire un fantasme d'élus. Les tenants de la centralisation, qui se sont tus pendant si longtemps relèvent la tête, à la faveur de dégradation de la situation économique et des comptes de la France et considèrent, à la limite la notion de " pouvoir local » comme un dangereux oxymore.

Il était temps de redéfinir une politique territoriale, non pour étatiser et recentraliser mais pour tenter de rendre plus performantes les institutions locales. L'examen attentif des mesures prises sous les quinquennats Sarkozy et Hollande montre qu'elles vont dans le bon sens et méritent d'être amplifiées et valorisées

Le cadre constitutionnel complété par Jean-Pierre Raffarin est tout à fait satisfaisant ; le principe de libre gestion des collectivités locales, dans leur sphère de compétence naturellement, posé par l'art.72 n'est pas incompatible avec un pilotage de l'ensemble " collectivités locales » au niveau national plus conforme aux possibilités de l'économie. Il appartient au Gouvernement et au Parlement de faire évoluer les choses. Aucune autre réforme constitutionnelle ne paraît nécessaire.

La liberté de gestion s'applique à chaque collectivité mais les associations de collectivités sont des groupes de pression comme les autres dans la mesure où elles émargent au budget de l'État.

La décentralisation reste une idée neuve en France. Le neveu de Chateaubriand, A. de Tocqueville, a bien montré dans l' "Ancien régime et la Révolution », que la centralisation, portée à son point culminant par Napoléon, traversait l'histoire de France. Pourtant, I'existence de larges responsabilités locales est nécessaire à l'épanouissement d'une démocratie authentique. La plupart des pays européens qui nous entourent sont plus décentralisés que nous ; la Grande-Bretagne, modèle du self-government, qui avait pris le chemin inverse vient d'adopter une loi de décentralisation!

À la pleine liberté d'entreprendre, principe révolutionnaire promu par le constituant de Rennes, le Chapelier, mais constamment rogné jusqu'ici, doit correspondre une grande liberté-responsabilité des collectivités locales : les libertés économiques et locales se complètent.

Cela dit, quatre actions mériteraient d'être menées avec persévérance:
- Le succès des métropoles et de nouvelles communautés de communes est d'ores et déjà perceptible. Le Grand Lyon, Nice, Toulouse, Rennes, Brest, notamment, sont bien partis.

À Aix-Marseille ce sera plus difficile. À Paris, on ne doit pas se satisfaire d'une métropole croupion : il faudra reprendre le dossier.

Aux élections de 2020, il faudra veiller à ce que les exécutifs communautaires et métropolitains soient effectivement élus au suffrage universel direct, afin d'assurer leur légitimité par rapport aux communes. Au sein du «bloc communal», la primauté des métropoles et des nouveaux groupements découlera de leur élection au suffrage universel direct; les conseils municipaux perdraient leurs pouvoirs fiscaux et les budgets communaux ne seraient plus que des enveloppes budgétaires arrêtées au niveau communautaire.

- La dévitalisation des départements est inévitable ; elle est effective dans le périmètre du Grand Lyon; elle va se réaliser sans bruit dans les autres métropoles; le transfert, un moment prévu, des transports routiers et ferroviaires au niveau régional devra être repris. La fusion entre les départements et la région a failli aboutir en Alsace ; cette solution doit être encouragée partout où elle est possible. La diversité des formules ne fait plus peur, depuis notamment l'érection du Grand Lyon en collectivité territoriale de plein exercice; pourquoi pas Nice, Marseille-Aix et bien sûr Paris. La clarification des compétences, objectif raté de la loi NOTRe, passe par l'intégration des départements pour partie dans les métropoles, pour une autre dans les régions. La carte des nouvelles régions a été dessinée un peu vite; ce qui compte c'est la volonté de vivre ensemble. Une Bretagne à cinq départements est souhaitée par les intéressés.

- Les ressources des régions ne sont pas à la hauteur de leurs responsabilités actuelles, ni des développements futurs; avec la fusion des départements et des régions, ces dernières recevraient les trois quarts de la nouvelle taxe sur la valeur ajoutée des entreprises! La réforme des ressources régionales ne pourra pas attendre longtemps.

- Enfin, il paraît souhaitable de prolonger au-delà de 2017 « l'association des collectivités territoriales à l'effort de redressement des finances publiques ", au rythme décidé par la loi de programmation 2015-2017 (-3,55 Md€ par an). Malgré les apparences, ce n'est pas contradictoire avec le développement des responsabilités locales, mais il faudra faire évoluer la méthode. Le rationnement purement comptable, concevable au départ, devra faire place à une approche plus intelligente. 
Poids respectif des trois catégories de collectivités territoriales (bloc communal, départements, régions)

\begin{tabular}{lcccccc}
\hline & \multicolumn{2}{c}{$\begin{array}{c}\text { Dépenses } \\
\text { de fonctionnement }\end{array}$} & \multicolumn{2}{c}{$\begin{array}{c}\text { Dépenses } \\
\text { d'investissement }\end{array}$} & \multicolumn{2}{c}{$\begin{array}{c}\text { Dépenses } \\
\text { totales }\end{array}$} \\
\hline & $\begin{array}{l}\text { Montant } \\
\text { (en Mde) }\end{array}$ & $\begin{array}{l}\text { Evolution } \\
\text { annuelle }\end{array}$ & $\begin{array}{c}\text { Montant } \\
\text { (en Mde) }\end{array}$ & $\begin{array}{l}\text { Evolution } \\
\text { annuelle }\end{array}$ & $\begin{array}{r}\text { Montant } \\
\text { (en Mde) }\end{array}$ & $\begin{array}{l}\text { Evolution } \\
\text { annuelle }\end{array}$ \\
\hline & & & & & & \\
Bloc communal & 89,3 & $+2,1 \%$ & 41,2 & $-9,6 \%$ & 130,6 & $-1,9 \%$ \\
Dé partements & 59,3 & $+2,8 \%$ & 14,2 & $-4,2 \%$ & 73,5 & $+1,4 \%$ \\
Régions & 17,8 & $+1,4 \%$ & 11,4 & $+2,8 \%$ & 29,3 & $+1,9 \%$ \\
& & & & & & $-0,4 \%$ \\
\hline Ensemble & 166,5 & $+2,3 \%$ & 66,8 & $-6,5 \%$ & 233,4 & $-0,4$ \\
\hline
\end{tabular}

Donnees hors gestion active de la dette.
Source : DGFP.

Évolution des dépenses locales en longue période

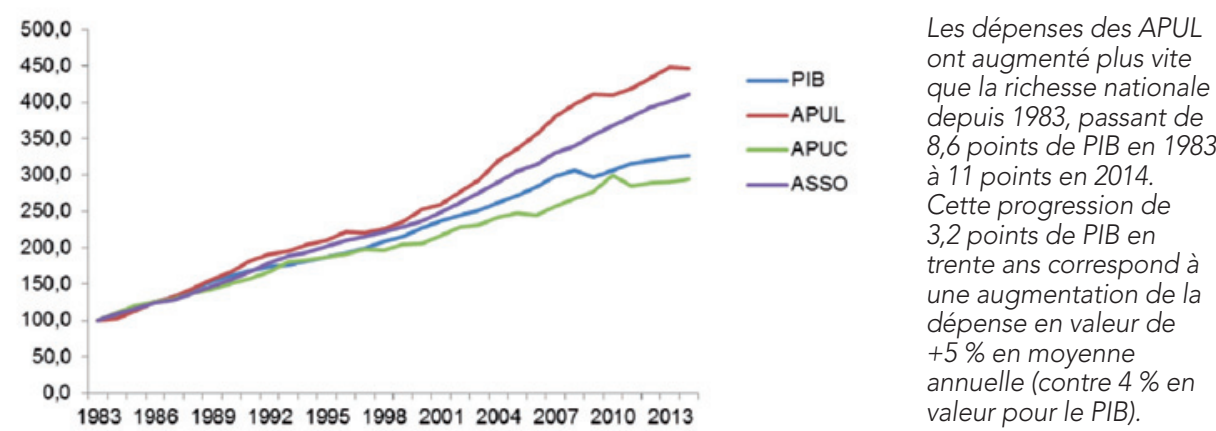

Les transferts financiers de l'État aux collectivités territoriales

\begin{tabular}{|c|c|c|c|}
\hline & 2015 & 2016 & Variation \\
\hline Total des transferts : $(1+2+3)$ & 101,5 & 99,5 & $-2,0 \%$ \\
\hline 1- Concours aux CL & 53,6 & 50,8 & $-5,3 \%$ \\
\hline 1.1- Prélèvements sur recettes État & 50,7 & 47,0 & $-7,3 \%$ \\
\hline dont DGF & 36,0 & 33,2 & \\
\hline 1.2- Autres concours & 2,9 & 3,7 & $28,0 \%$ \\
\hline 2- Impôts d'État transférés & 31,3 & 31,7 & $1,5 \%$ \\
\hline $\begin{array}{l}\text { dont : } \\
\text { 1- } \text { Acte } 1 " \text { Décentralisation }\end{array}$ & & & \\
\hline 2.1- «Acte 1 "Décentralisation & 9,8 & 10,1 & $2,3 \%$ \\
\hline 2.2- « Acte $2 "$ (Raffarin) & 12,3 & 12,6 & $2,0 \%$ \\
\hline 2.3- Réforme Fiscalité 2000 & 7,1 & 7,1 & $0,1 \%$ \\
\hline 3- Formation Professionnelle & 2,8 & 2,8 & $-0,1 \%$ \\
\hline
\end{tabular}

En 2016, le montant total des trans ferts de l'Etat aux collectivités locales est passé sous la barre des $100 \mathrm{Md} €$, soit globalement : $-2 \%$.

La ponction a porté sur les prélèvements sur recettes budgétaires de I'État: $-7,3 \%$. Une réduction de même montant sera opérée en 2017.

\section{Sources}

— Travaux législatifs : www.senat.fr/territoires/index.html

— Site Internet «Intérieur, Finances » : www.collectivites-locales.gouv.fr

- L'Observatoire des finances locales, 19 e rapport (2015) : www.collectivites-locales.gouv.fr/rapports-lobservatoire-desfinances-locales-ofl

- Le « jaune » budgétaire 2016, présentant l'évolution des finances locales depuis 30 ans et les conditions de «l'association » des collectivités locales au redressement financier du pays ».

www.performance-publique.budget.gouv.fr/sites/performance_publique/files/farandole/ressources/2016/pap/ pdf/jaunes/jaune2016_collectivites.pdf 